ENCYCLOPÉDIE Encyclopédie berbère

BERBERE

$27 \mid 2005$

27 | Kairouan - Kifan Bel-Ghomari

\title{
Khanguet Si Mohamed Tahar
}

(Aurès) site néolithique. Aux origines du pastoralisme berbère

\section{Roubet}

\section{OpenEdition}

\section{Journals}

Édition électronique

URL : http://journals.openedition.org/encyclopedieberbere/1350

DOI : 10.4000/encyclopedieberbere. 1350

ISSN : 2262-7197

Éditeur

Peeters Publishers

Édition imprimée

Date de publication : 1 août 2005

Pagination : 4205-4228

ISBN : 2-7449-0538-0

ISSN : 1015-7344

Référence électronique

C. Roubet, " Khanguet Si Mohamed Tahar », Encyclopédie berbère [En ligne], 27 | 2005, document K52, mis en ligne le 01 juin 2011, consulté le 25 septembre 2020. URL : http://journals.openedition.org/ encyclopedieberbere/1350; DOI : https://doi.org/10.4000/encyclopedieberbere.1350

Ce document a été généré automatiquement le 25 septembre 2020.

(c) Tous droits réservés 


\title{
Khanguet Si Mohamed Tahar
}

(Aurès) site néolithique. Aux origines du pastoralisme berbère

\author{
C. Roubet
}

\section{Topographie-toponymie}

1 Bien au-delà de Constantine*, après avoir traversé le bassin de Timgad* en direction du versant septentrional de l'Aurès*, s'ouvre à l'ouest une voie d'accès au massif, le Foum Ksantina ([Fum Qsen,ina]). Elle emprunte le défilé du Khanguet Si Mohamed Tahar ([Xanget Si Muiemmed a aher], ici KSMT), où coule le torrent mugissant Berbaga. Ce torrent qui se jette dans l'oued Taga occupe une position géographique symétrique de celle de l'antique Abigas*, oued Bou Roughal, ou Baghaï* à l'est du massif. C'est en suivant cette vallée qu'on entre vers $1000 \mathrm{~m}$ d'altitude dans un amphithéâtre ceinturé de parois calcaires de plus de $500 \mathrm{~m}$ de hauteur, culminant à $1874 \mathrm{~m}$ vers l'est, au Djebel Témagoult, et à $1540 \mathrm{~m}$ vers l'ouest, au Crêt et au plateau du Lardam (Redam ou Rdam). Ce plateau est traversé par la route nationale reliant Batna à Biskra. Cette toponymie est portée sur les cartes géologiques et IGN au $1 / 200000^{\mathrm{e}}$ et au $1 / 50000^{\mathrm{e}}$. Dans ce paysage s'est ébauché, voilà presque 7500 ans cal. BP, un comportement pastoral ayant favorisé l'enracinement d'un premier peuplement semi-sédentaire, dont les Berbères Chaouia* descendent très vraisemblablement.

\section{La grotte Capéletti du Khanguet Si Mohamed Tahar}

2 Cet amphithéâtre verdoyant parait fermé de toutes parts. Mais on peut en sortir en suivant en amont le torrent jusqu'à sa source résurgente au sud-est, puis, en se glissant dans le passage argileux, encombré de gros blocs d'où jaillissent les jets d'eaux d'une ligne de sources. On aperçoit alors les ruines du tout premier moulin* à grains édifié vers 1900 par le premier meunier de la région, le Piémontais Jean-Baptiste Capéletti (Roubet, 1969, 1979 ; fig. 2-3). En grimpant encore dans les éboulis de la rive gauche, on débouche enfin sur le plateau du Lardam cerné au loin de murailles enneigées d'où émergent le Djebel Mahmel (2 $321 \mathrm{~m}$ ). Le regard découvre alors un vaste paysage mis en 
cultures. Bien plus près, on remarque à $50 \mathrm{~m}$ environ du sommet de l'amphithéâtre, un énorme effondrement calcaire figé sur la pente de la rive gauche du torrent Berbaga. Cette masse diaclasée, détachée de la muraille, s'est enfoncée dans les éboulis (fig. 4). Ses pans abrupts tournés vers la vallée présentent de petites ouvertures ainsi qu'une grotte nichée à l'avant d'un couloir couvert d'un toit. Cette grotte dite tantôt du Foum Ksantina, tantôt du Khanguet Si Mohamed Tahar porte depuis 1969 le nom de Capéletti, en hommage à la perspicacité et à l'activité de cet émigré, amoureux d'une région qu'il contribua à développer. C'est lui qui fit connaître cette grotte néolithique au géologue R. Laffitte (Joleaud et Laffitte 1934) et qui remit les premiers objets préhistoriques recueillis au début du $\mathrm{xx}^{\mathrm{e}}$ siècle, mêlés au guano de la grotte qu'il exploitait alors. Il s'agissait de haches et d'herminettes en pierre polie, d'instruments en os poli, de pièces en métal, abandonnés par les divers occupants. Ces objets furent déposés à Paris, au Musée de l'Homme, où ils sont toujours conservés au département de Préhistoire du Muséum national d'histoire naturelle (réf. Collection. 36.1) (fig. 6 et 7).

Fig. 1. Grottes et abris néolithiques du Maghreb oriental connus en 1968.

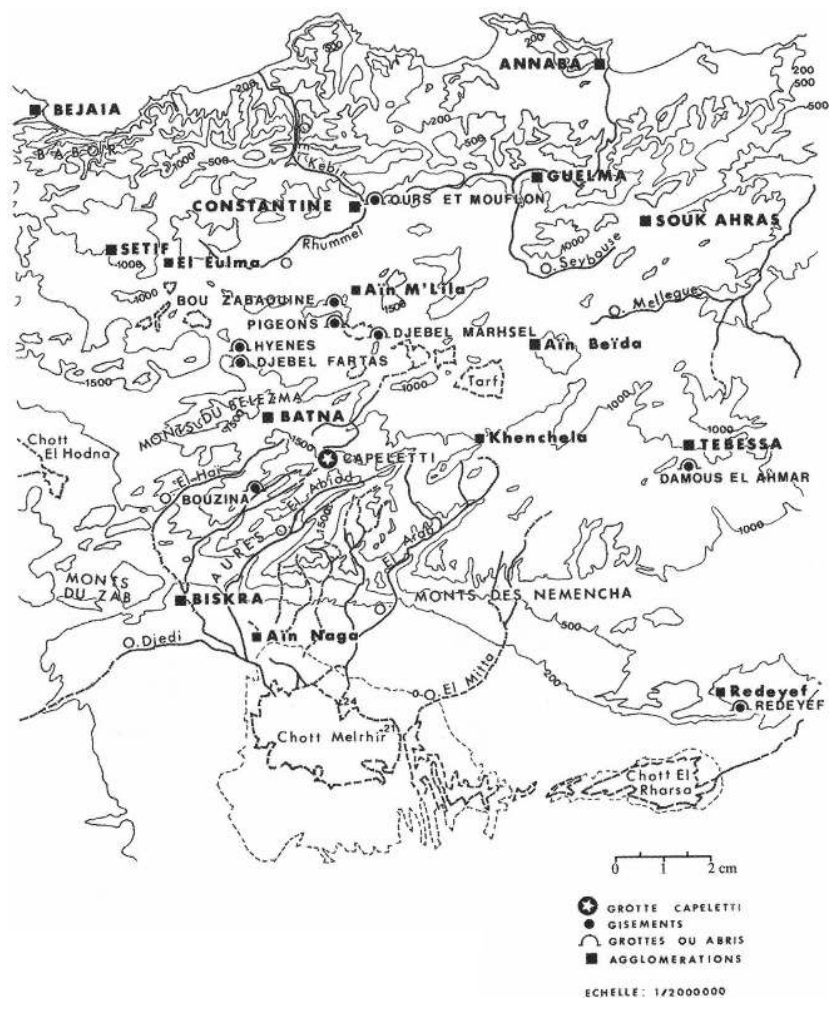


Fig. 2. Jean-Baptiste et Hemana Capéletti en 1971 devant leur maison de Bou Ahmar, Aurès (cl. B. Ferré, mars 1971).

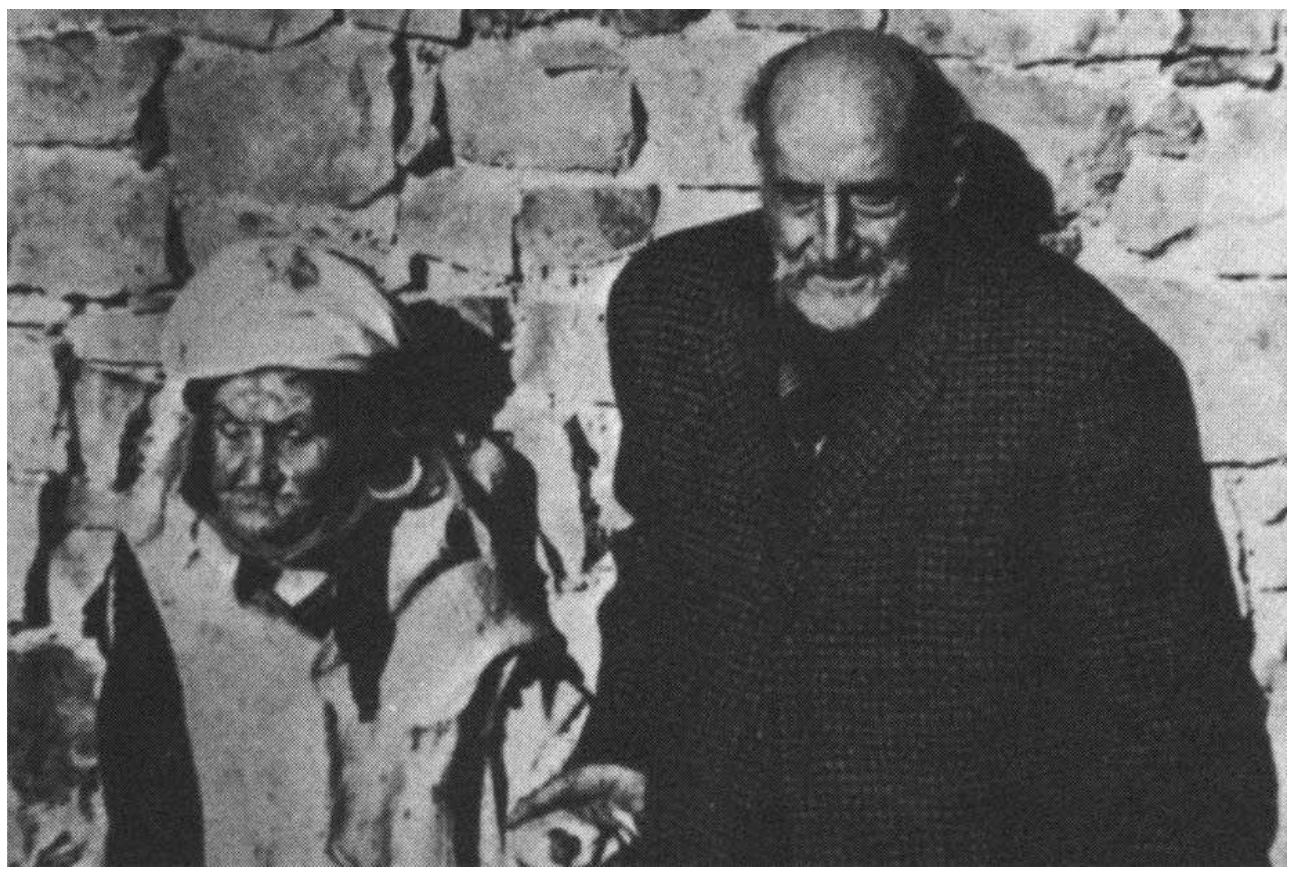

Ce territoire a été un conservatoire exceptionnel. Il a fait vivre et s'épanouir pendant des millénaires de multiples communautés pastorales qui ont façonné les paysages alentour. Nous pensons même qu'un proto-commerce s'est ébauché là. En témoignent quelques documents néolithiques d'origine exogène, conservés parmi d'autres, fabriqués sur place. Ce sont des haches, des herminettes, des éléments de parure, etc. Tous accréditent l'hypothèse $d^{\prime}$ '« acquisitions » lointaines faites au fil du temps, lors des transhumances annuelles que des bergers néolithiques inaugurèrent à travers ce massif. Au retour de leurs déplacements, bêtes et gens regagnaient un territoire montagneux familier et s'installaient avec "leur trésor » dans cette grotte, résidence d'été, grenier à provisions, lieu d'agnelage protégé, permettant à tous de goûter la quiétude et les ressources locales. Le massif de l'Aurès garde une mémoire vive de ce lointain passé pastoral (Roubet 2003a).

\section{Grotte CAPÉLETTI -DATATIONS 14C -CALIBRATION (Prog. Cal 98)}

\begin{tabular}{lcccccccccc}
\hline Laboratoire & Localisation & Profondeur & Nature & $14 \mathrm{C} / \mathrm{BP}$ & $14 \mathrm{C} / \mathrm{BC}$ & Millénaires & Cal BP & Cal BC & Milléénaires & Occupations \\
\hline Alg.0037 & B3a & $3,17-3,14$ & charbons & $6530 \pm 250$ & 4580 & V & $6859-7855$ & $5906-4910$ & VI-V & $1^{e c}$ \\
\hline Gif.1386 & Alf & 2,00 & charbons & $5900 \pm 150$ & 3950 & IV & $6400-7032$ & $5083-4451$ & VI-V & $2^{e}$ \\
\hline Gif.1383 & B3a & $2,95-3,00$ & charbons & $5740 \pm 140$ & 3800 & IV & $6279-6807$ & $4858-4330$ & V & $2^{e}$ \\
\hline Gif.1384 & B1f & $1,00-1,10$ & charbons & $5400 \pm 140$ & 3450 & IV & $5894-6453$ & $4504-3945$ & V-IV & $3^{e}$ \\
\hline Gif.1385 & A1h & $1,62-1,40$ & charbons & $5380 \pm 140$ & 3430 & IV & $5889-6447$ & $4498-3940$ & V-IV & $3^{c}$ \\
\hline Alg.0062 & J3i & $1,25-1,40$ & charbons & $4360 \pm 130$ & 2410 & III & $4777-5316$ & $3367-2828$ & IV-III & $4^{e}$ \\
\hline Alg.0030 & D3f & $0,35-0,40$ & charbons & $4340 \pm 200$ & 2390 & III & $4414-5471$ & $3522-2465$ & IV-III & $4^{e}$ \\
\hline
\end{tabular}


Fig. 3. À gauche, vallée encaissée du Berbaga : le premier moulin de Capéletti est construit sur la rive droite du torrent. À droite, J.-B. Capéletti devant la grotte en compagnie de sa femme et de sa chienne Dékha (cl. R. Lafitte, 1934).
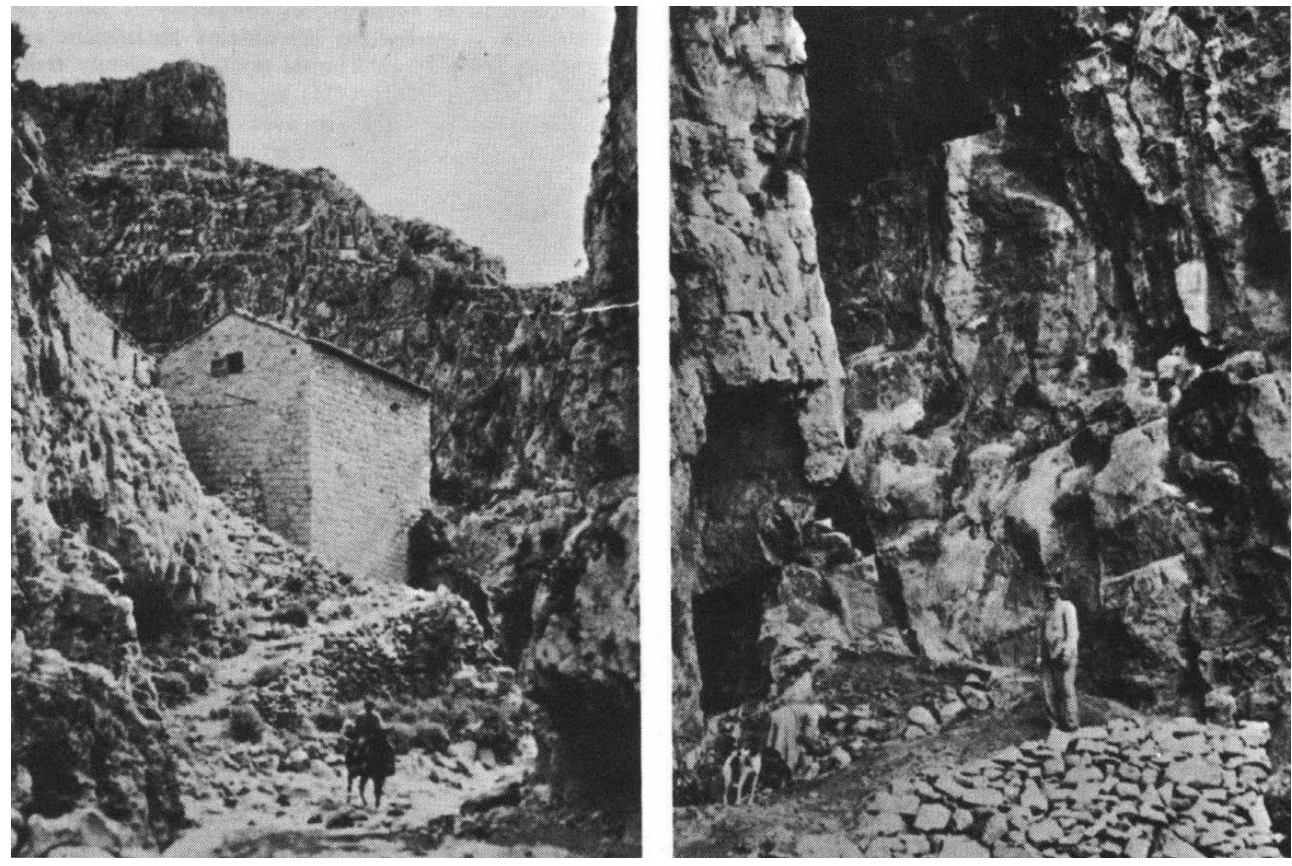

Fig. 4. Vue aérienne signalant l'emplacement de la grotte Capéletti dans le bloc calcaire diaclasé. A gauche, s'étend la plaine du Lardam, à droite, coule le torrent Berbaga (cl. B. Férré, mars 1971).

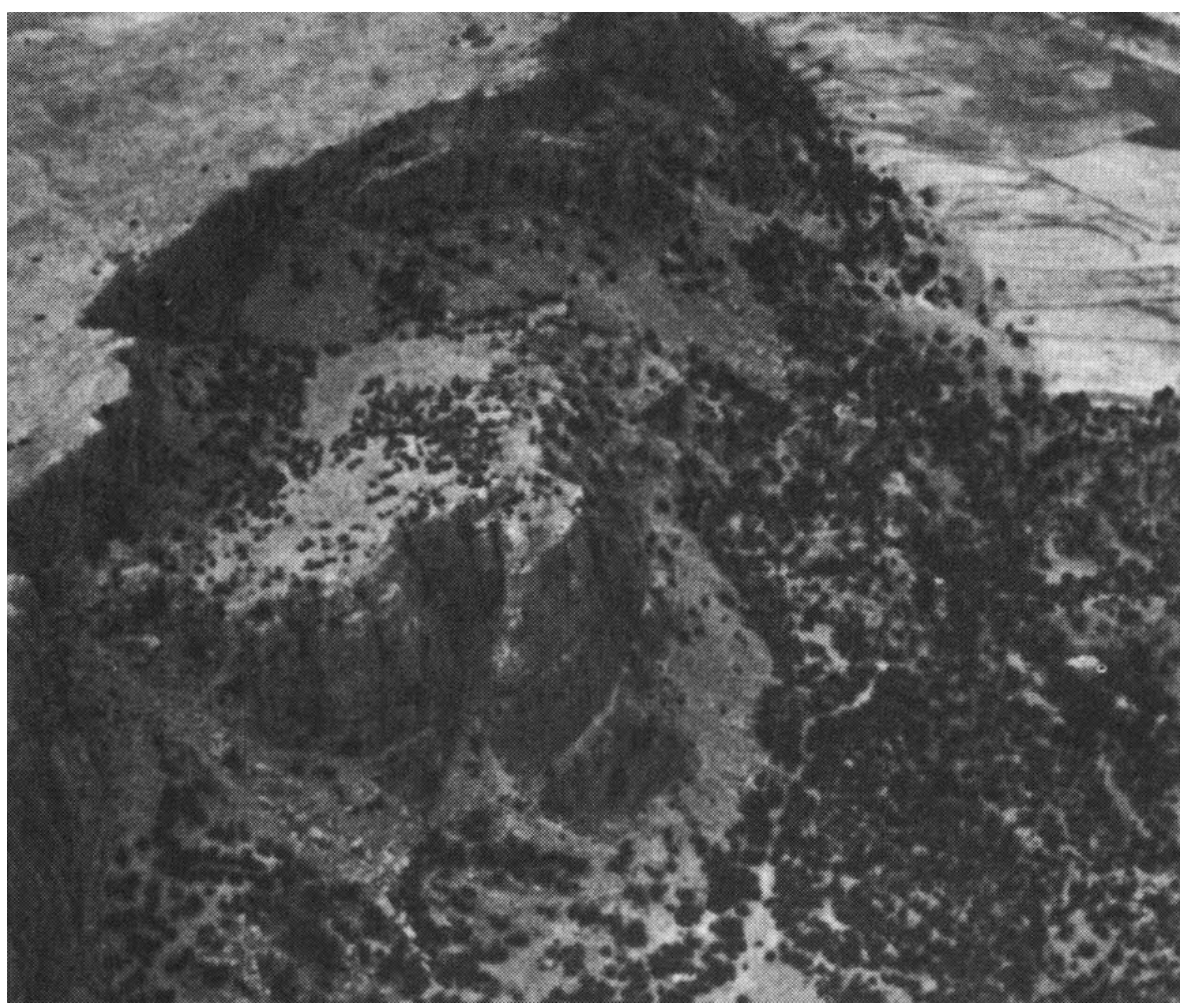




\section{Vers une perception globale du comportement pastoral néolithique}

4 A la suite de deux campagnes de prospection du massif, puis de fouilles conduites dans cette grotte, l'une dirigée en 1934-1936 par Thérèse Rivière et Germaine Tillon, accompagnées de Jacques Faublée, anthropologues du Musée de l'Homme de Paris, l'autre en 1968-1970 par Colette Roubet, attachée de recherche au Cnrs, affectée au Crape à Alger, une documentation abondante a été réunie. L'une est conservée à Paris, l'autre à Alger; les deux se complètent. L'occupation néolithique traitée dans une optique interdisciplinaire -géologique, topographique, chronologique, sédimentologique, écologique, anthropologique et culturelle - a révélé l'installation d'une succession de communautés pastorales, dont l'histoire a été partiellement retracée (Roubet 1979 ; Bachir-Bacha 1996).

Fig. 5. Levé Topographique de la grotte Capéletti effectué en 1968, les hachures couvrent l'aire fouillée. Échelle 1/100 (relevé de J.-L. Paillet).

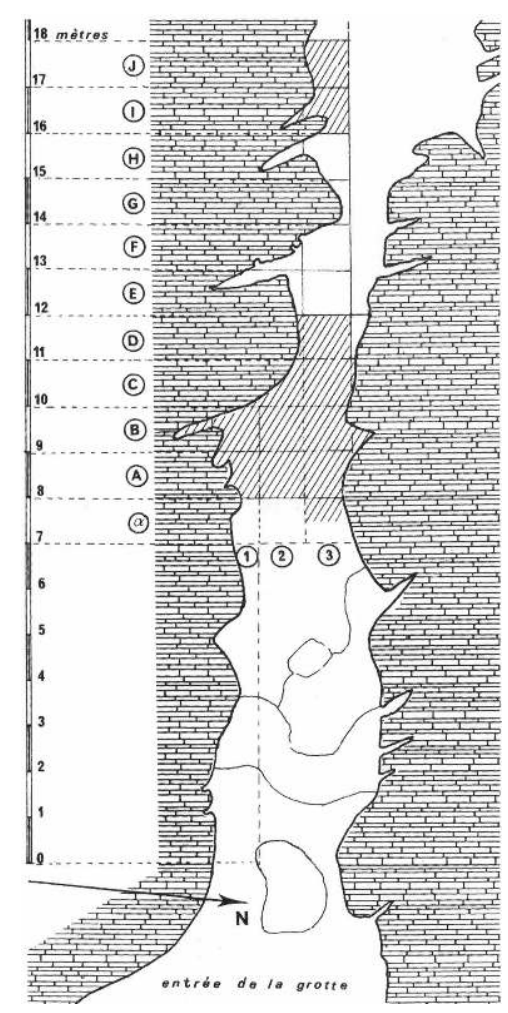


Fig. 6. Haches polies et piquetées découvertes par Th. Rivière dans les dépôts supérieurs " $A$ ». Documents déposés au Musée de l'Homme, Paris (cl. J.-P. Kauffman). $\mathrm{N}^{\circ} 1: 36$. 1. 1987. Hachette plate, polie $\mathrm{N}^{\circ} 2: 36$. 1. 1030. Hache cylindrique, piquetée $\mathrm{N}^{\circ} 3: 36.1$. 1968. Hachette plate, polie $N^{\circ} 4: 36$. 1. 1931. Hache cylindrique, piquetée.

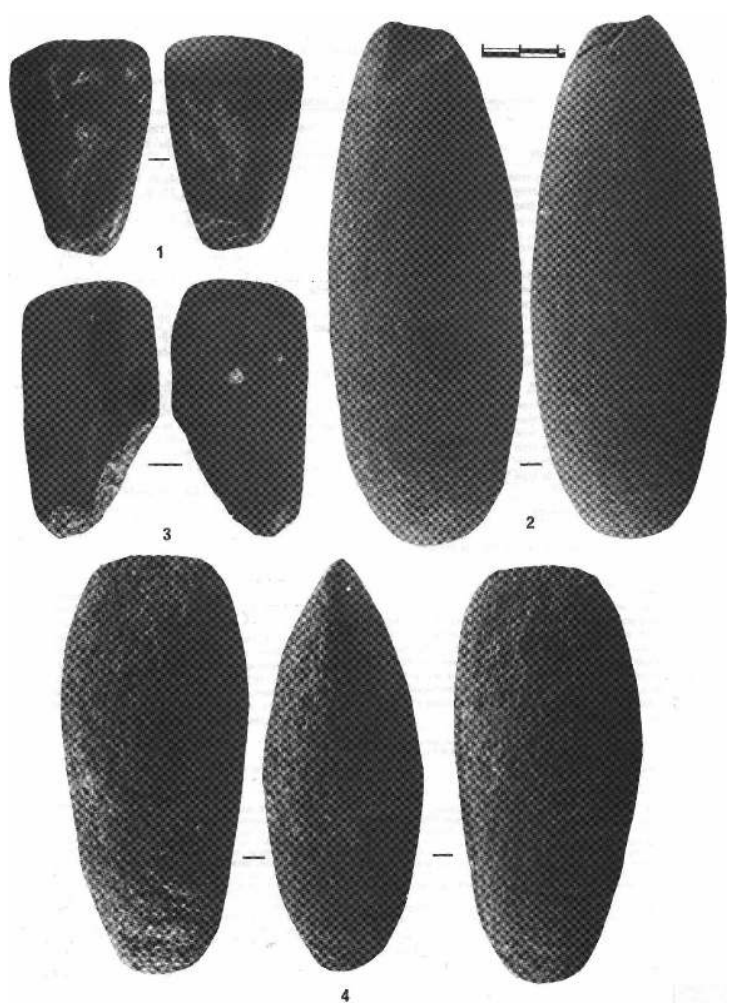

5 Au cours du VIII ${ }^{\mathrm{e}}$ millénaire cal. BP, un petit groupe de bergers trouve refuge à $1540 \mathrm{~m}$ d'altitude dans la grotte Capéletti (fig. 1, 3, 4 et 5). Il s'installe au printemps sur les flancs du Khanguet Si Mohamed Tahar, avec moutons et chèvres et peut-être déjà une paire de bœufs ( ?). De ce bref séjour subsistent de multiples objets quotidiens et des restes d'une nourriture variée et locale, jetés au fond d'un chaos rocheux de la grotte.

Entre 7 500-5 000 cal. BP, bien d'autres générations de bergers viennent occuper ce site de façon saisonnière. À l'intérieur de la grotte, leurs déchets comblent ce chaos qui sert de dépotoir. Dès la fin de l'automne, bêtes et gens quittent régulièrement ces lieux lorsque d'abondantes chutes de neige bloquent les passages et tarissent les ressources. Les bergers partent en transhumance et gagnent alors des zones plus clémentes de piémont (Roubet 1979, 1984, 1985).

7 Mais que sait-on de ces grands départs, du séjour hivernal vécu ailleurs? Reste-il quelque chose encore de l'histoire de ces multiples transhumances? Il fallait faire le tour d'une réalité humaine facettée, à peine entrevue en 1979, pour voir surgir quelque chose d'un vécu pastoral total du premier peuplement semi-sédentaire pré-berbère de l'Aurès (Roubet 1995, 2003(a), 2003(b)). La démarche a consisté à valoriser tout ce qui venait d'ailleurs :

- partir du support insolite et rare, du caractère exogène du document, privé de son cortège d'éléments de fabrication et de ses références techniques,

- partir des documents botaniques et fauniques, étrangers à l'environnement en altitude,

- circonscrire des aires géographiques précises, des biotopes spécifiques, à partir de la provenance identifiée, et peut-être retrouvée, pour chaque document. 


\section{Le terroir montagnard originel : le KSMT et l'Aurès pendant l'holocène}

8 Les données stratigraphiques et chronologiques du remplissage de la grotte Capéletti permettent une lecture bimillénaire continue, sans horizon stérile. Ce remplissage a été attribué au Néolithique de Tradition Capsienne sensu stricto, faciès régional, porteur d'une forte valeur identitaire. On situe ainsi ses principaux épisodes :

- la ${ }^{\text {re }}$ installation des bergers remonte au VIII ${ }^{\mathrm{e}}$ millénaire cal. BP, entre 5906-4910 BC.

- la $2^{\mathrm{e}}$ série d'occupations se prolonge, entre les VIII-VII ${ }^{\mathrm{e}}$ millénaire cal. BP, entre 5083-4451/4858-4330 BC.

- la $3^{\mathrm{e}}$ série d'occupations se situe au VIII ${ }^{\mathrm{e}}$ millénaire cal. BP, entre 4504-3945/4498-3940 $\mathrm{BC}$.

- la $4^{\mathrm{e}}$ et dernière série d'occupations se situe, au $\mathrm{VI}^{\mathrm{e}}$ millénaire cal. $\mathrm{BP}$, entre 3367-2828/3522-2465 BC.

9 La grotte cesse ensuite d'être la résidence des pasteurs de cette culture-là. On ignore tout des motivations de l'abandon; on ignore presque tout des occupants postérieurs.

10 Au terme d'une longue évolution, ces générations de bergers détiennent avec leurs troupeaux un tel capital économique qu'ils doivent attirer la convoitise de groupes ou de communautés non pastorales qu'ils rencontrent, et s'imposer aussi à eux en raison de l'identité et de la forte cohésion communautaire qu'ils affirment. Un statut de berger leur conférant une forte autonomie devait déjà leur être reconnu. Parvenue à un stade d'évolution économique avancé, cette communauté néolithique s'est approprié, bien plus que le Khanguet Si Mohamed Tahar et sa grotte-résidence: le massif de l'Aurès tout entier. Et cela, plutôt sans partage, ni pression extérieure. Pourtant, c'est soudainement ici que s'arrête leur histoire, nous ne sommes plus en présence de vestiges pouvant leur être attribués. 
Fig. 7. Herminettes et haches polies, découvertes par J.-B. Capéletti Documents déposés au Musée de l'Homme, Paris (cl. J.-P Kauffman). 1 : Herminette, réf. 36.1.82 ; 2 : Herminette, réf. 36.1.84; 3 : Herminette, réf. 36.1.81; 4 : Hache, réf. 36.1.2307 (ou 36.1.83 pour Th. Rivière).

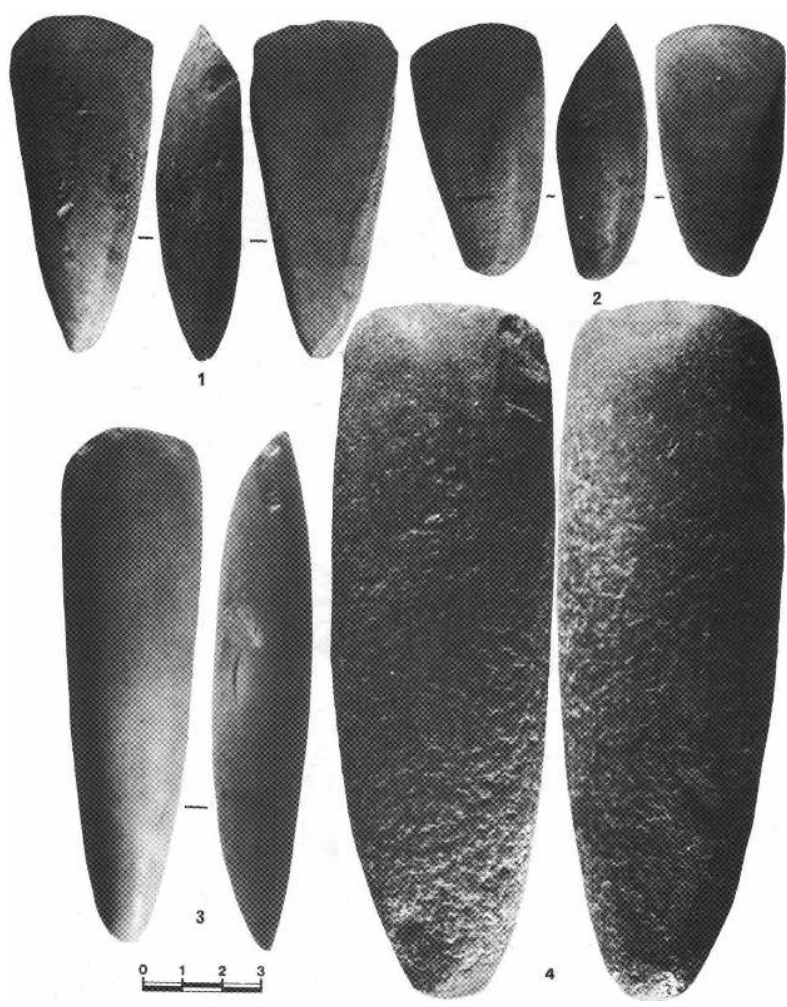

11 Précisons maintenant les conditions climatiques et écologiques des séjours des bergers en altitude entre $7500-5000$ cal. BP. Les données climatiques et environnementales autorisent l'identification de deux épisodes majeurs (Alimen, Biéda, Casta 1979). D'autres événements d'âge historique affecteront le massif (Ballais 1987; Côte 1987, 1991).

Entre le VII-VI ${ }^{\mathrm{e}}$ millénaire cal. BP, sévit vers $2000 \mathrm{~m}$ d'altitude un climat très froid et sec, devenant humide ( $1^{\mathrm{er}}$ épisode), propice au développement d'essences à caractère «boréal » telles : Corylus (le noisetier) ; Carpinus (le charme), Ulmus (l'orme), Betula (le bouleau) et Eleagnus (Beucher 1979). Vers $1500 \mathrm{~m}$, règne aussi un climat rude et humide, favorable à l'if, installé près du torrent (niche à Taxus baccata) et au développement d'essences à caractère méditerranéen, telles le cèdre, certains chênes, les frênes dimorphes, les thuyas, les genévriers, l'oléastre et le noyer.

u milieu du VI millénaire cal. BP, lorsque la rigueur s'atténue, d'autres frênes et des pins croissent sur les pentes, ainsi que la vigne sauvage, le sorbier, les phillyrées, le prunus, l'arbousier, le lierre, l'asphodèle, les roseaux, tout un cortège d'herbacées temporaires occupant les sous bois, pelouses et prairies. Cet épisode du milieu de l'Holocène est rattaché en Afrique du Nord à l'étage climatique de montagne méditerranéenne, de type marocain (Couvert 1969 ; Beucher 1979 ; Plu et Portères 1979) (fig. 9).

Durant le $\mathrm{V}^{\mathrm{e}}$ millénaire cal. BP s'opère un réchauffement climatique ( 2 e épisode) entraînant le recul des mousses et des lichens fixés aux rochers et favorisant une abondante fructification. Les grappes de raisin sauvage, les arilles rouges des ifs et les glands doux (Quercus ilex, var. ballota) du chêne ballotte, sont les fruits souvent cueillis 
et rapportés en grotte. Fait alimentaire capital: la glandée automnale assure les premières semoules. Un goût de farine reste associé aux lieux. Une dizaine de fruits secs ou juteux, doux ou acides, sont attestés parmi les macro-restes végétaux conservés. D'autres témoins : feuilles, hampes, racines, bois, évoquent des collectes sélectives, renouvelées, destinées à une alimentation animale, à l'aménagement du couchage, à une médecine empirique et à des activités supposées de tressage, tissage, vannage, teinture, en plus de préparations alimentaires, exigeant combustible, chaleur et lumière (Couvert 1969, Plu 1979, Portères 1979).

Plusieurs études paléobotaniques permettent la reconstitution d'un couvert végétal restituant la profusion de ce terroir, près des hauts sommets, couverts par la cédraie et la thuriferaie, adaptées aux froids et à l'enneigement prolongés. On admet actuellement un découpage altitudinal du massif en cinq géo-systèmes (Ballais 1984, 1987).

Dans leur diversité et leur amplitude, les multiples résidus botaniques collectés évoquent tous des gestes intentionnels de préhension, d'expérimentation, de manipulation et de fabrication.

17 Le cadre environnemental de l'Aurès à l'Holocène se révèle très proche de celui d'autres montagnes méditerranéennes.

\section{Vers une anthropisation progressive de l'Aurès durant l'Holocène}

18 L'anthropisation du Maghreb oriental s'est amorcée durant l'Épipaléolithique. Mais c'est plutôt au début de l'Holocène que s'installent loin de la mer et dans des campements distincts, des individus issus des deux communautés autochtones d'alors. L'une, composée de Cromagnoïdes-Méchtoïdes évolués, est imprégnée depuis longtemps de culture ibéromaurusienne (Hachi, 1998, 1999, 2002; Roubet et Hachi 2001b, 2003) ; l'autre, constituée de Proto-Méditerranéens, initie une culture capsienne. Elles occupent le Tell, puis les Hautes Plaines du Constantinois ainsi que la périphérie des dépressions lagunaires ou Garaet et Sebkhas, cernant de petits chaînons du système atlasique.

Des individus de ces deux communautés se trouvent même exceptionnellement réunis dans certains gisements-sépultures, comme par exemple à Médjez II, de faciès Sétifien, (Camps-Fabrer 1975). Les restes anthropologiques de Proto-Méditerranéens sont toutefois plus nombreux dans les escargotières (Camps 1974). On admet que l'exploration de l'Aurès a été conduite par ces deux populations et qu'elle coïncide avec l'Holocène. Mais aucune datation actuellement connue ne remonte à cette époque là. 
Fig. 8. Objets de parure du IVe millénaire BC.

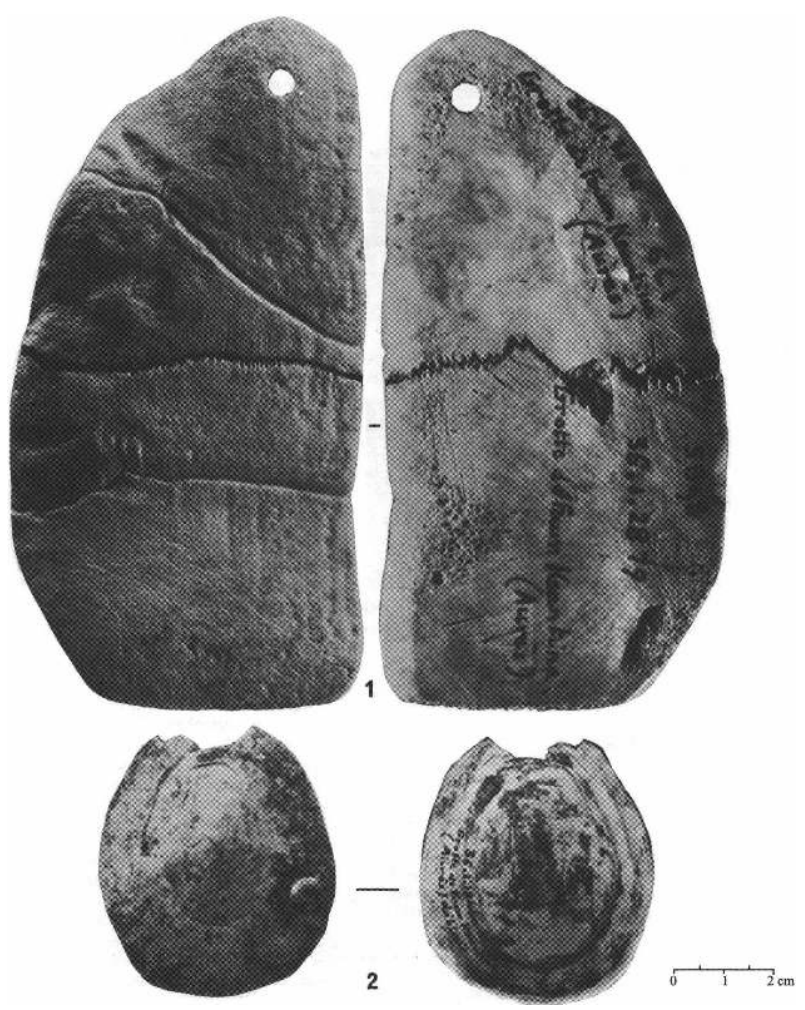

Le grand pendentif taillé dans deux plaques du plastron d'une tortue terrestre a été découvert par Th. Rivière, scindé en deux parties ; la partie supérieure provient de la couche « $C$ » et la partie inférieure de la couche «B ». Ce n'est qu'en 1970 que je les ai rapprochées et replacées en connexion

anatomique (Roubet 1979, fig. 48). La patelle, d'origine méditerranéenne, a été découverte par J.-B. Capéletti. Elle contient des colorants rouges et des sédiments mêlés. Ces documents sont déposés au Musée de l'Homme à Paris (cl. J.-P Kauffman).

Dans le massif aux traits géomorphologiques puissants et originaux (Mittard 1941), la localisation systématique des installations épipaléolithiques reste à poursuivre. Quarante sites ont été inventoriés. Cette situation lacunaire ne donne pas une image significative. Il s'agit d'escargotières capsiennes (Ballais et Roubet 1981-1982 ; Roubet 1979).

21 Au nord du massif, autour des sebkhas du Tarf, du bassin du Hodna, puis vers Ouled Djellal et Ouled Naïl, enfin près des grands Chotts, se groupent des sites capsiens datés (IX ${ }^{\mathrm{e}}$ millénaire cal. BP ; Grébénart 1969, 1970, 1971 ; Camps 1974, 1975).

Antérieurement au Néolithique, certains versants extérieurs de l'Aurès avaient donc été occupés durant l'Épipaléolithique.

C'est vers le milieu de l'Holocène que l'anthropisation s'est étendue à tous les massifs du Constantinois, de Tébessa et de l'Aurès, se manifestant même en altitude. Cette anthropisation a été conduite par les deux populations déjà implantées comme dans la grotte du Dj. Fartas (Roubet 2001a), ou celle du Damous el-Ahmar, prés de Tébessa, qui comprenaient des individus de type Méchtoïde évolué (Roubet 1968, 1979, 2001a). Toutefois, les motivations de cette pénétration dans les massifs n'étaient plus celles de chasseurs-nomades opportunistes, Capsiens ou autres, mais désormais celles de bergers engagés dans un projet pastoral sans précédent. 


\section{Le projet pastoral des bergers du Néolithique de tradition capsienne (NTC) du KSMT}

Le projet pastoral couvre la période $7500-5000$ cal. BP. Initié par des bergers NTC, il a consisté à maintenir en équilibre, pendant les quatre séries d'occupation de la grotte, l'accroissement des troupeaux et le développement des communautés humaines, au cœur d'un terroir familier et humanisé. Au Khanguet Si Mohamed Tahar cet objectif a concerné des communautés successives, composées de 7 à 15 individus et de 15 à 40 bêtes.

Ce projet a pris délibérément en compte le cycle annuel des saisons et les ressources disponibles en altitude et sur les piémonts de l'Aurès. Pour maintenir la cohésion et l'épanouissement de chaque unité, des transhumances annuelles ont été organisées, reliant les zones d'estivage en altitude aux zones d'hivernage sur les piémonts.

Le déroulement de ce cycle n'a pas conduit le groupe humain à se replier, ni à vivre en autarcie; il ne l'a pas culturellement figé. Au contraire, on remarque l'introduction dans l'habitat estival d'éléments étrangers rares, suivie de l'entrée régulière d'autres biens d'importance. Toutes ces innovations durent concourir à modifier progressivement le contenu culturel initial du NTC en interférant sur la mentalité symbolique collective. Ces documents exogènes apparaissent donc comme des témoins privilégiés d'une acculturation progressive, collective et régulée (Roubet 1979: chap. 7-9).

27 En somme, si la culture NTC initiale et son projet pastoral ont pu évoluer sans soubresaut, semble-t-il, mais par greffes progressives de nouvelles valeurs, ne serait-ce pas en raison du rôle majeur que les bergers purent faire jouer à leurs troupeaux et surtout de la dynamique qu'ils purent leur impulser? Si l'on peut proposer ce schéma d'évolution des comportements, c'est parce que l'archéologue pense qu'il ne put en être ainsi qu'au prix d'un consensus communautaire, d'un accroissement des troupeaux, d'un approvisionnement toujours soutenu en biens exogènes, équitablement répartis. Hypothèse notamment suggérée par l'accroissement continu des troupeaux et des biens, durant toutes les occupations. 
Fig. 9.1 a, Taxus baccata L. ; b, niveau B1, 140-150 cm ; 2 a, Juniperus oxycedrus L. ; b, niveau B1, 130-140 cm ; 3 a, Pinus pinaster Soland. ; b, niveau BI, 130-140 cm ; 4 a, cupule de Quercus llex L. ; $b$, niveau B1, 130-140 cm ; 5 a, Quercus Ilex L. ; b, niveau B2, 140-150 cm ; 6 a, Vitis sylvestris L. ; b, niveau A2, 150-160 cm ; 7 a, Sorbus torminalis Crantz ; b, niveau B1, 170-180 cm ; 8 a, Prunus prostrata Labill ; b, niveau B2, 140-150 cm ; 9 a, Papaver Rhoeas L. ; b, niveau A2,150-160 crn ; IQ a, Phillyrea angustifolia L. ; b, niveau A2, 125-140 cm ; 11 a, Lygeum Spartum L. ; b, niveau A2, 160-180 cm ; 12 a, Vicia villosa Roth ; b, niveau B2, 250-270 cm (cl. A. Plu, mars 1973).

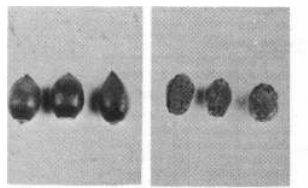

1 a
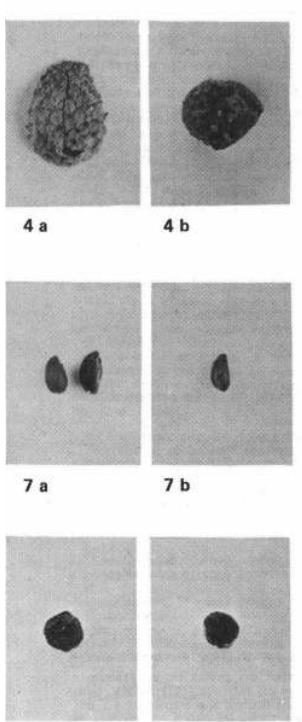

10 a

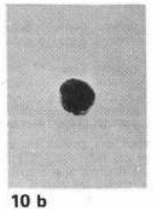

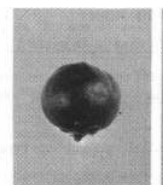

2 a

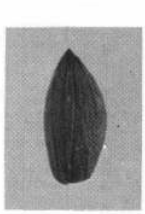

5 a
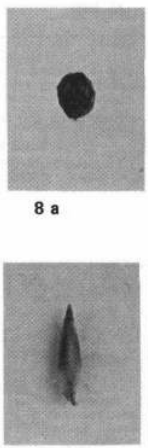

11

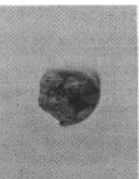

$2 \mathrm{~b}$

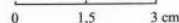

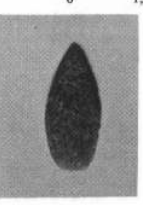

$5 \mathrm{~b}$

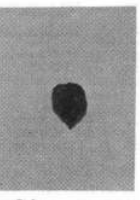

$8 \mathrm{~b}$

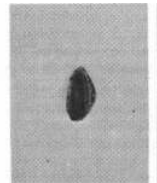

3 a

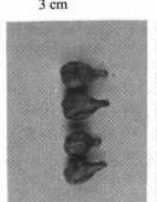

6 a
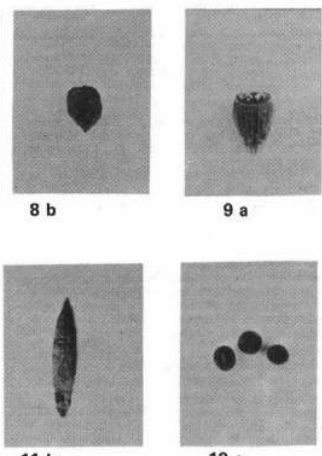

12 a
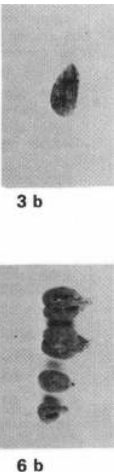

$6 \mathrm{~b}$
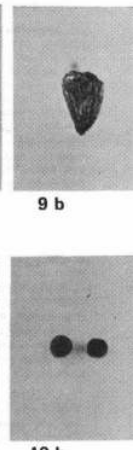

L'évolution de ce projet est constatée vers la fin de la première installation des bergers en grotte ; elle se traduit par une ouverture sur le "monde extérieur " à l'Aurès que les transhumances favorisent, et par l'entrée en grotte de nouveaux documents. Cette ouverture est restée compatible avec:

- le maintien d'une identité pastorale et d'une cohésion de l'unité communautaire,

-l'accroissement des troupeaux, comme valeur de capital et d'épargne et parfois d'échange,

- le contact avec des colporteurs, non pasteurs, détenteurs de biens de prestige hautement spécialisés, diversifiés et renouvelés,

- un acte d'échange, faisant suite à une transaction, établissant la parité des produits convoités : biens culturels rares pour les uns, biens alimentaires rares pour les autres, reposant sur un engagement réciproque de la parole donnée,

- le principe d'une interdépendance des objectifs et des décisions concernant l'élevage, le mode de gestion des troupeaux, la consommation et l'acquisition de biens culturels exogènes,

- la diffusion de ce genre de vie pastoral et la dispersion de nouvelles communautés pastorales.

Afin de valider ces différents points, on examinera à travers les diverses activités, les témoignages d'une acculturation et d'une anthropisation progressives. 


\section{Estivage et hivernage au Khanguet Si Mohamed Tahar : territoires et documents}

\section{La grotte-grenier-résidence d'été}

Ce projet a permis à chaque unité communautaire de séjourner au KSMT pendant 6 à 8 mois, du printemps à l'automne. L'unité s'est servie de la grotte Capéletti comme d'abri fixe et de base de regroupement; chaque communauté en a disposé sans partage, pleinement, comme d'un "grenier-résidence ", qui prit au fil du temps le caractère d'une "propriété communautaire ». Ce trait déjà singulier tient à la difficulté de son accessibilité. Ce lieu, très retiré dans la partie nord du massif, est resté bien dissimulé dans la falaise diaclasée durant deux millénaires. Son éloignement des terres de parcours et sa localisation protectrice ont été des arguments décisifs lors du choix de cet abri. Dans l'amphithéâtre, la grotte occupe enfin une position privilégiée à l'adret, sous les crêtes, à l'abri des rigueurs septentrionales et proche des berges du Berbaga. Dans les alpages alentour cernés par d'épaisses forêts, bêtes et gens ont séjourné en toute quiétude, à l'écart d'observation et de visites étrangères. Ce qui subsiste du vécu de ces petites collectivités successives paraît diversifié et récurrent; cela évoque un genre de vie « équilibré », autosuffisant, organisé. L'intérieur exigu de la grotte n'a pas reçu de modifications. L'entrée tournée vers l'est, donne accès à deux salles, peu éclairées, parfois ensoleillées une heure ou deux pendant la belle saison (Ferré 1979); elles ont été occupées sans aplanissement $d u$ sol, après stabilisation des rochers. L'effondrement de plusieurs masses rocheuses a marqué la deuxième série d'occupations (Roubet 1979, 2001a). D'autres petits éboulements ont affecté les derniers dépôts. Un couloir sombre, temporairement occupé, s'étire sur 21m (Paillet 1979).

\section{Aménagement et entretien de l'espace ; ébauche d'un réseau de sentiers}

Autour de la grotte-nid d'aigle, quelques espaces ont été occupés après défrichement, empierrement et soutènement; ce sont (fig. 4) : une plate-forme, un replat, un accès aux points d'observation au-dessus de la grotte, un chemin sûr dans les éboulis; un enclos à bétail, un gué.

Ces espaces immédiats sont devenus indispensables pour assurer le déroulement quotidien d'activités diverses. De nos jours, les versants sont zébrés par des sentiers longeant crêtes, pentes et berges, qui quadrillent l'espace (Roubet, 2001a).

Dans la prise en compte de l'étendue du territoire d'altitude investi par les premiers pasteurs, il faut ajouter l'espace des troupeaux au pâturage, sur les versants du KSMT et le plateau du Lardam. Dans cette perspective, l'entretien d'un réseau de sentiers devait être accompli collectivement. Cet aménagement général de l'espace a toujours été assuré en période estivale, par piétinement, cheminement et broutement des troupeaux, ainsi que par rejet, amoncellement des détritus et défrichement occasionnel.

Tous ces faits indirects ayant conditionné le séjour des pasteurs viennent renforcer l'hypothèse d'un début d'anthropisation du Khanguet Si Mohamed Tahar, délibérément organisé (2-3-4 éries d'occupation). 


\section{Témoins locaux de l'estivage}

\section{$1^{\text {re }}$ série d'activités : aménagement des foyers, approvisionnement, transport et transformation (salles, plate-forme)}

Aucune structure de foyer remontant aux premières occupations de la grotte n'a été repérée. Après l'hivernage précédant la $2^{\mathrm{e}}$ série d'occupation, des aires de foyers ont dû être ré-agencées et approvisionnées. En témoignent des charbons de bois brûlés, des pierres craquelées, des récipients brisés et divers détritus rejetés dans la fosse dépotoir de la $2^{\text {e }}$ salle (Rivière, 1935-1936; Roubet 1979 : chap. 6-10). Un seul type de foyer s'est conservé inscrit en cuvette et sans empierrement dans les sédiments meubles des dernières fréquentations de la grotte (Roubet 1979). Bien qu'approprié à des préparations culinaires d'appoint, à feu doux, régulier, ce foyer pourrait n'avoir pas été adapté à la cuisson du gros gibier (exiguïté du couloir, manque de dégagement, d'aération, d'évacuation des fumées). À l'extérieur, la localisation d'autres emplacements de foyers reste inconnue.

\section{Nourritures en relation avec des récipients et des instruments (salles, plate- forme)}

Les récipients (cuisson, stockage) n'étaient jadis ni rangés, ni vides. On n'a pas observé d'espace de rangement permanent, à l'écart du foyer et de la poubelle, ni d'anfractuosité dans la paroi, pourtant une relation spatiale rapproche les marmites des lieux de combustion. Trois catégories de récipients ont été identifiées : les grandes bouteilles céramiques à fond conique (hauteur $35 \mathrm{~cm}$, contenance 4,5 litres); d'autres petites bouteilles correspondent au remploi d'une coquille entière d'œuf d'autruche (1,5 litres); de grandes marmites céramiques à fond sub-conique (hauteur $25 \mathrm{~cm}, 5 / 6$ litres), sans bec, mais pourvues d'une large ouverture $(25-30 \mathrm{~cm})$ et de moyens de préhension, d'autres petites marmites de forme ovoïde (1,5 litres); des bols et des coupelles ( 0,5 litre) taillés dans de grandes portions de coquille d'œuf d'autruche, ou dans des boucliers dermiques de tortue terrestre, enfin des outres en peau, non conservées.

Les instruments de cuisine associés aux récipients et aux nourritures pourraient avoir servi à trancher et couper (couteaux, racloirs, éclats, lames, scies); à trouer et perforer (perçoirs, poinçons, becs); d'autres à gratter et écharner (grattoirs, objets à extrémité mousse, denticulés) ; enfin, les derniers destinés à broyer, écraser et réduire en menus morceaux ou en poudre (meules, molettes, broyeurs). Légers, les outils tranchants en silex taillé et en os taillé-poli se trouvaient à côté de résidus de viandes consommées ( $2^{\mathrm{e}}$ série d'occupation) (Rivière 1934-1936 ; in Roubet 1979 ; in Bachir-Bacha 1996). Entiers, ces outils ont pu avoir été oubliés et sont alors tombés au fond de la poubelle. Vers l'entrée de la grotte, dans la paroi calcaire gauche, à un mètre au-dessus du sol actuel, existent des incisions fusiformes, proches des "traits capsiens", témoins d'âge incertain de l'affûtage du tranchant des couteaux.

Les ossements abandonnés près des braises (grillades) sont ceux de moutons, chèvres, bovins et autres animaux chassés ou piégés (tableau 1 ). Au cours de la $3^{\mathrm{e}}$ série d'occupation les bergers ont aussi consommé des escargots cuits. On a retrouvé des lits épais de coquilles entières colorées, parfois brûlées. La fosse conservait plusieurs 
centaines de noyaux de fruits, des graines, des glands doux grillés et oubliés. Ces derniers, récoltés en quantité (3-4 ${ }^{\mathrm{e}}$ séries d'occupation), avaient été stockés non loin de pièces de broyage (Roubet 1979).

\section{Préparation et entretien du mobilier utilitaire (salles, plate-forme extérieure)}

La grande panoplie d'outils lithiques taillés a été créée, affinée, réparée et avivée in situ, comme le suggèrent nucléus et déchets divers. Mais on n'a retrouvé ni emplacement, ni équipement (billot, enclume) liés à cette activité. Les roches taillées ici ne sont pas locales. Le silex fait défaut. Cette matière première a donc toujours été introduite dans la grotte. On peut en déduire une gestion et une maintenance des outils accomplies avec plus de vigilance : grattoirs et perçoirs ravivés. La grande panoplie d'outils et objets osseux polis fait suite à une consommation carnée estivale, un stockage, puis une sélection des pièces anatomiquement les plus appropriées (industrie osseuse).

Une gamme d'instruments lithiques lourds, destinés au broyage, a été façonnée à partir de roches locales : calcaire, quartzite, grès, poudingue. Il s'agit de billots, meules, molettes et broyeurs, de conception simple, d'aménagement sommaire, présents dès la $2^{e}$ série d'occupation. Citons enfin d'autres documents lithiques polis, introduits en grotte déjà façonnés, parfois en calcaire, mais surtout en silex et roches basiques exogènes, comme la diorite, la micro-diorite et la serpentine (Bachir-Bacha 1996). Il s'agit de haches et d'herminettes.

\section{Préparation et entretien d'éléments de protection et d'embellissement}

41 Les objets évoquant une fonction prophylactique (animale, humaine) et d'embellissement corporel, rattachés à une parure, sont nombreux et divers. Ils ont été fabriqués à partir de matériaux locaux ou exogènes (présence de déchets et pièces cassées), sans bénéficier d'emplacement réservé. L'attestent divers os longs d'oiseaux, des tests d'œuf d'autruche, du derme ossifié de plastron de tortue conservant jadis son écaille, des coquilles, des colorants d'hématite rouge et de la galène. Ces supports ont permis la création d'éléments tubulaires gravés, de grains d'enfilage circulaires ou dentelés et de larges pendentifs, tous, brillants et teintés. Dans le plastron de plusieurs tortues terrestres (testudo ibera, cf. mauritanica), des pendentifs ont été créés. Techniquement standardisés, morphologiquement définis, reproduits en nombre, prisés par les pasteurs, ils ont connu une large diffusion à travers le Maghreb atlasique (Roubet 1966, 1968), favorisée peut être par l'extension du pastoralisme (fig. 8).

\section{Préparation des peaux, assemblage et confection diverses}

42 À l'aide d'outils lithiques (grattoirs, racloirs, denticulés, perçoirs) et d'outils en os poli (brunissoirs, spatules, aiguilles), les peaux ont dû être traitées, puis assemblées.

\section{$2^{\mathrm{e}}$ série d'activités : transport, transformation de produits alimentaires frais et rares}

43 La plupart des produits carnés frais proviennent de l'élevage et du piégeage. Les activités de boucherie liées à la fabrication de l'outillage osseux, devaient être très précisément codées. 


\section{Activités communautaires, près de la grotte}

Certaines activités ponctuelles devaient être effectuées collectivement. L'abattage d'un ovin, d'un caprin, ne fut pas un acte fréquent, ni un événement banal. On pourrait suggérer la pratique d'un "rituel » antérieur à l'abattage (présence d'un couteau-lame de racloir). Dans les dépôts rien n'atteste un abattage en nombre, au mieux un abattage circonstanciel, réduit à quelques individus. Les restes osseux issus des fouilles de la $\mathrm{l}^{\text {re }}$ salle n'étaient pas abondants (Bachir-Bacha 1996). Ceux de la $2^{\mathrm{e}}$ salle totalisent 12050 pièces, dont 10407 pièces d'ovi-caprinés et 1355 pièces de bovins (tableau 1). Les données fauniques ont été établies par P.-L. Carter et E.-H. Higgs (1979) :

- $\mathrm{l}^{\text {re }}$ occupation : $89,7 \%$ d'ovins-caprins (4 individus), et 7,3\% bovins ( 1 individu), $-2^{\mathrm{e}}$ occupation : $84,7 \%$ d'ovins-caprins ( 20 individus), et $14,0 \%$ bovins ( 6 individus), - $3^{\mathrm{e}}$ occupation : $90,4 \%$ d'ovins-caprins (30 individus), et $6,8 \%$ bovins ( 3 individus), - 4 e occupation : $70,0 \%$ d'ovins-caprins ( 4 individus), et $24,7 \%$ bovins ( 1 individu).

Ces données quantitatives apparaissent aujourd'hui comme partielles. En effet, elles compriment de multiples faits d'abattage et de consommation, survenus au cours de plusieurs épisodes d'estivage, rattachés par défaut à une même série d'occupation.

Ces données ne sont représentatives que d'une certaine consommation prise en grotte (tableau 1).

Rappelons aussi que ces données sont privées d'un certain nombre de pièces anatomiques, prélevées par les bergers pour le façonnage de leurs outils en os poli. Enfin, à aucun moment R-L. Carter et E.-S. Higgs ne tentent de donner des estimations des troupeaux d'animaux vivants. Il convient de rappeler l'esprit dans lequel ce travail pionnier a été entrepris en 1970-72 et de le valoriser ici encore, puisqu'il reste sans égal à ce jour en Algérie et étaye ici l'idée d'une pastoralisation en évolution.

4 Voilà donc atteint le point de départ d'une relecture des informations fauniques permettant de faire participer le troupeau tout entier à la dynamique générale du projet pastoral, en montrant que celui-ci dut être aussi perçu aussi comme une composante non alimentaire.

\section{Activités individuelles ou collectives, programmées au KSMT par les pasteurs}

rtunistes et parfois individuelles, ces activités ont introduit en grotte divers produits alimentaires obtenus par piégeage et captures d'une part ; ce sont surtout des lièvres, hérissons, tortues, batraciens, oiseaux (2-3-4e séries d'occupation); et d'autre part, lors de chasses aux prédateurs, ce sont des sangliers et marcassins $\left(1^{\mathrm{e}}\right.$ à $4^{\mathrm{e}}$ séries d'occupation avec une plus forte proportion au cours de la $3^{\mathrm{e}}$, et des chacals $\left(3^{\mathrm{e}}-4^{\mathrm{e}}\right.$ séries d'occupation) (Carter et Higgs 1979).

Après les pluies, d'autres activités à caractère collectif ont été programmées. D'amples ramassages de gastéropodes ont été pratiqués, comme l'attestent les nappes de coquilles comprenant Leucochroa candidissima, Hélix melanostoma, Hélix punica, Helicella sitifensis, Hélix aspersa, et Rumina decollata (Roubet 1979).

D'abondantes cueillettes devaient se dérouler sur les pentes et le plateau. Celles de fruits mûrs, de graines, de champignons (non attestés), puis des récoltes de tubercules et de racines, avant l'émondage des arbres (fourrage), la coupe des fougères (litières); le prélèvement de gommes, résines et miels dans de petits récipients (Beucher 1979, Plu 
1979, Portères 1979). En automne, des récoltes intensives de glands doux, particulièrement attendues, devaient être accomplies collectivement.

D'autres collectes plus rares ont procuré d'insolites documents locaux, comme ce moule interne d'oursin fossile d'Heterodiadema libycum (Joleaud et Laffitte, 1934; Laffitte, 1939).

\section{Témoins lointains de l'hivernage prélevés sur les piémonts et près des lagunes}

Les témoins lointains sont parfois exogènes au massif. Nombreux, divers, lourds, ou légers et fragiles, transportés et introduits en grotte, ils se sont étroitement mêlés aux documents de l'estivage, jusqu'à les rejoindre plus tard dans la poubelle. Issus de délocalisations, certains, comme les végétaux évoquent des actes intentionnels de préhension dans quelques cas et, d'autres en revanche fortuits. On note aussi la présence de biens culturels rares et insolites dont la conception est originale. Ces biens mettent en valeur des matériaux exceptionnels, venus d'ailleurs eux aussi, y compris de la mer.

Nous distinguerons donc deux classes de biens : les uns prélevés dans l'environnement par les bergers et les autres, issus d'acquisitions probables par échanges.

\section{Approvisionnement en roches à tailler : piémont sud du massif}

$\mathrm{Au}$ KSMT, l'absence de formations contenant des nodules de silex a exigé la découverte de gîtes donnant des roches à tailler, de bonne qualité. Cette carence majeure, une fois surmontée, a pu lors des remues déterminer la direction des parcours et fixer par avance les lieux de haltes. L'objectif de prélèvement et de transport de roches à tailler fut donc impérativement pris en compte par toutes les générations de bergers. Certains silex étaient noirs, gris et beige, mais de petites dimensions $(\mathrm{L}<10 \mathrm{~cm})$, d'autres blonds et de grandes dimensions $(\mathrm{L}>12 \mathrm{~cm}$ ). L'attestent des résidus d'encroûtement de formations nummulitiques conservés sur le cortex de produits d'épannelage, qui incitent à localiser sur le piémont sud la provenance probable du silex, vers Rhoufi, M'Chounèche, au Dj. Rhéliss et au Kroumt el-Kreloua entte Menâa et Bouzina (Laffitte, 1939 et com. pers.). La strontianite des calibreurs affleurerait aussi à Rhoufi (Laffitte, 1939 et comm. pers. ; Roubet, 1979).

\section{Alimentation en territoire d'hivernage : Zones marécageuses des piémonts nord ou sud}

Compte tenu des caractéristiques (altitudinales et paléo-environnementales) des territoires fréquentés l'hiver et de leurs biotopes, l'alimentation des pasteurs et des troupeaux devait être variée et relevée. Des bribes de cette nourriture ne pouvaient évidemment parvenir en grotte qu'après intervention, délibérée ou fortuite, humaine ou/et animale (transport). Parmi les éléments qui ont subsisté, certains proviennent de denrées de route tardivement consommées, jetées dans la poubelle en arrivant dans la grotte (végétaux et ossements d'antilope et de gazelle). D'autres permettent d'identifier et de localiser les zones fréquentées. 

(Alimen, Biéda et Casta. 1979). D'importants gîtes de sel en plaques existent vers le sud, au Djebel Mélah, à El Outaya, lieu d'exploitation séculaire (Ballais 1987), et vers le nord, là où s'étendent les zones salines des petits Chotts du Tarf. A l'occasion des prélèvements de sel, des récoltes intentionnelles de végétaux halophiles ont été pratiquées. Il s'agit d'Atriplex halimus et des salicornes, du sparte (Lygeum Spartum), pour le tressage de nattes et de filets (non présents) et de l'alfa (Stipa tenacissima), pour la préparation de vanneries (non conservées) (Plu et Portères 1979). D'autres encore, comme Ziziphus lotus et Pistacia atlantica, provenant d'une brousse à jujubier, voisine des dépressions salées (Beucher 1979), suggèrent des haltes alimentaires coïncidant avec la maturation des fruits (résidus conservés). Une florule zoochore, composée de plantes héliophiles et nitratophiles, pourrait bien avoir été fortuitement transportée dans les toisons du cheptel, puis s'être introduite en grotte par hasard, avant de se mêler aux sédiments. Bien que peu nombreux, ces documents végétaux sont désormais parmi les témoins les plus précieux de la grotte. Eux seuls « disent » l'importance alimentaire de l'acte de préhension pratiqué en zone sub-saline. Eux aussi évoquent le moment dans la saison et le séjour au bord des lagunes. Seuls ces végétaux témoignent enfin sans ambiguïté de la fréquentation par les pasteurs d'espaces non sécurisés qu'à leur époque d'autres individus nomades ne traversaient peut être pas. En somme, seuls des bergers vigilants conduisant leurs troupeaux sur ces terrains là, ont pu transporter sur eux, à leur insu, tout comme leur cheptel, graines et fruits nitratophiles de ces lointaines contrées.

Dans ces plaines et basses terres lagunaires parcourues par les autruches, les bergers ont prélevé et consommé les œufs dénichés. Des dizaines de coquilles vides mais entières d'œuf d'autruche ont été rapportées en grotte, avec précaution, pour servir de bouteilles, puis de petits récipients. Dans les clairières, ils ont aussi capturé de nombreuses tortues terrestres pour satisfaire une consommation immédiate, mais ont su conserver et transporter en grotte les carapaces entières, non brisées, pour les transformer ensuite en bols, puis en pendentifs. De la chasse aux Antilopinés (antilope) et aux Oryginés (gazelle), il subsistait au total 52 restes osseux d'une nourriture de retour, conservée dans la grotte (tableau 1).

Compte tenu de la diversité et des ressources de ces paysages, de la proximité de ces nombreux biotopes, près des piémonts nord et sud du massif, on ne s'étonnera ni de la fréquence des actes de prédation durant l'hivernage, ni d'une très large exploitation des ressources faciles à capturer, pêcher ou collecter, sous le couvert végétal collinaire, le long des ruisseaux, des fleuves, tout autour des marais et des lagunes salées.

Ceci conduit à reconnaître durant l'hivernage, à travers ces actes prédateurs fréquents et diversifiés, le retour à des pratiques et à des comportements de nomades temporaires.

\section{Acquisition par échange de biens culturels d'importance : vers le nord}

61 L'impact de l'introduction en grotte de biens culturels exogènes au massif ayant déjà été souligné (Roubet 1979), il reste à apprécier ici l'éventuel retentissement de ces biens sur la communauté et ses traditions culturelles (Roubet 1995).

Encyclopédie berbère, 27 | 2005 


\section{Outillage ? Ou bien objets spéciaux en pierre polie ? Introduits en grotte} maîtrise et sobriété (par qui, et où ?) dans une roche énangère au massif (venant d'où ?), au VIP millénaire cal. BP. Comment considérer cette pièce et les suivantes lorsque manque tout ce qui parle de fabrication? Matières premières, outils, déchets? Lorsque l'usage et la destination, restent inconnus, quel statut, quel rôle peut-on leur reconnaître?

Au Maghreb, l'âge d'apparition de ce matériel et de la technologie complexe mise en œuvre, restent très incertains. On soupçonne que ce nouveau concept d'instruments lithiques polis était acquis en Algérie dès ix ${ }^{e}$ millénaire cal. BP. Mais d'où provenaient ces objets, par qui furent-ils façonnés? Comment s'intégrèrent-ils aux contextes culturels post-épipaléolithiques, comment a-t-on interprété leurs semblables provenant de la grotte de Brézina, près d'El Arouia-Dj. Amour, (F.-E. Roubet, in Camps 1974) ? Au Sahara central les repères manquent aussi, à l'exception de celui de MénietBaguena V, dans le Hoggar, qui remonte au vie millénaire cal. BP (Hugot 1963).

Sachant que tous les documents issus de la grotte Capéletti étaient achevés, l'hypothèse provisoirement retenue a été celle d'une fabrication extérieure, systématique, réalisée par des spécialistes, installés peut être dans la ou les région(s) d'origine de ce(s) matériau(x). Mais comment savoir si les objets introduits en grotte provenaient d'une source unique, ou bien de plusieurs, dépendantes à la fois de la provenance de la roche et du type d'instrument façonné ? Comment savoir si ces spécialistes devenaient à l'occasion colporteurs?

On sait d'un regard que ces objets lithiques polis sont typés, que leur corps est galbé, que leur biseau transversal est poli, qu'ils ont été préparés à partir de silex brun, gris et vert, de micro-diorites grises, de diorites vert-olive et grises, enfin de serpentine noire. Ces roches affleurent toutes vers le nord, dans le Tell Constantinois, entre Collo et le Cap Bougaroun pour la serpentine, dans le Djebel Nador et à Boudjoudoun près d'El Milia, pour les micro-diorites (Notices explicatives des Cartes géologiques de Constantine-Nord et Constantine-Sud, 1937).

- La première hache au corps cylindrique et piqueté, au biseau poli, est en micro-diorite grise ; elle est apparue au début de la $2^{\mathrm{e}}$ série d'occupation.

- Durant la $3^{e}$ série d'occupation, douze haches et herminettes entièrement polies ont été décomptées. Leurs types respectifs sont nets, leurs dimensions sont très variables. Leur poids varie entre $75 \mathrm{~g}$ pour une herminette et $400 \mathrm{~g}$ pour une hache, la plus lourde hache pesant $746 \mathrm{~g}$. Peu de biseaux ont été réaffûtés, quelques pièces sont endommagées, d'autres ont un biseau émoussé, mais la plupart sont presque intactes. Si bien qu'on ne peut pas soupçonner d'usage dur et violent.

- Quinze exemplaires de qualité proviennent des derniers dépôts et des déblais cumulés. On doit à J.-B. Capéletti d'avoir découvert la première très grande hache bouchardée et polie, en micro-diorite grise (réf. 36.1.2307) et la première herminette double, en silex gris-brun poli (réf. 36.1.81), dans les derniers sédiments néolithiques (Roubet 1979, coll. Musée de l'Homme, Th. Rivière, réf. 36.1.82, 83 et 84) (fig. 2, 6 et 7), tableaux 2-3.

Pourquoi tant de ces instruments pénétrèrent-ils là-haut? Pour tenter de comprendre les motivations des bergers, il convient de ne pas les confiner sur leurs terres 
d'hivernage, ni de dissocier ces objets inhabituels du cycle pastoral de ces populations. En réexaminant les produits exogènes ramenés en grotte après les transhumances des 2-3-4 séries d'occupation, dans une optique de convoitise entretenue par des colporteurs, on a pu noter un net accroissement du nombre des acquisitions et cru pouvoir proposer une corrélation entre ces témoins-là et une fréquence accrue des rencontres, celles-ci devenant de moins en moins fortuites. Dans cette optique, si haches et herminettes ne résultaient ni de vols ni de dons, mais plutôt de " transactions ", il a fallu que l'échange ait été équitable et qu'à l'objet convoité par les bergers corresponde une contrepartie de valeur également convoitée et appréciée du colporteur. Après avoir fait le tour des preuves tangibles, potentiellement attractives pour des nomades, l'hypothèse d'une denrée rare s'est imposée : un quartier viande de mouton ou de chèvre ou un animal entier, selon l'importance du troc.

Peut-on situer géographiquement l'échange ayant permis l'acquisition de haches et d'herminettes? - D'abord vers le nord, aux alentours de Timgad-Aïn M'Lila. Mais, nous verrons ci-dessous que pour d'autres documents exogènes, il faut se tourner plutôt vers le sud.

\section{Coquilles méditerranéennes, pièces en ivoire, colorants, introduits en grotte, après échange}

Associés aux deux dernières séries d'occupation, ces nouveaux documents durent participer aux compositions de protection et d'embellissement corporels. Certains ont été découverts par J.-B. Capéletti (fig. 8) (in Roubet 1979, fig. 39). Aucun ne pouvait avoir été récolté par hasard car il s'agit de pièces rares, délicatement façonnées, d'une haute valeur symbolique. Ce sont :

- 3 tubes de dentales; une patelle polie, formant coupelle avec des colorants; un segment de pétoncle. De provenance méditerranéenne, ces pièces ont pu pénétrer en région atlasique en contournant les massifs et en suivant les basses terres lagunaires septentrionales ou méridionales ;

- une perle ajourée, un fragment de bracelet, et un cylindre, en ivoire, objets remarquables de provenance plutôt septentrionale, comme l'attestent d'autres pièces en ivoire des grottes néolithiques du Constantinois ;

- des boules de colorants mats, d'hématite rouge, jaune, orange, et une plaquette brillante de galène (fard des yeux ou khôl), de provenance méridionale ; ces substances existent vers Taghit, près de Bouzina (Lartigue 1904).

69 A l'occasion de ce regroupement synchronique des objets, on voit se dessiner des cheminements aussi bien septentrionaux que méridionaux, apportant encore la preuve d'une succession de transhumances comprimées à l'intérieur d'une seule série d'occupation. Ce qui altère le degré de précision des informations.

$70 \mathrm{Au}$ terme d'un recensement des témoins majeurs d'estivage et d'hivernage, on perçoit mieux le rôle de réceptacle privilégié que la grotte Capéletti du KSMT a dû jouer. Sa position en altitude en fit une résidence-clé, protectrice, pour chaque communauté. Bien qu'elle servit l'hiver de remise aux outils lourds et aux récipients fragiles, on a pu lui reconnaître aussi une fonction de grenier à provisions, ancêtre possible de la guélâa de l'Aurès, et, en été, une fonction de bergerie, de lieu d'agnelage. Complexe et chargée d'une mémoire archéologique bien conservée, cette grotte a vu naître la vie et s'exprimer la joie. De là-haut, des communautés pastorales organisées partirent pour 
de lointaines expéditions, tournées aussi bien vers les territoires du Nord, contrastés et cléments, collinaires et lagunaires (d'où vinrent haches, herminettes, sel, coquilles marines, ivoire) que vers ceux du Sud, déjà présahariens, occupés par de grands Chotts (qui fournirent silex, sel, coquilles marines, colorants, galène) (Roubet 2001a, 2003b).

Tableau 1. Inventaire et répartition de la faune de la grotte Capéletti (Aurès, Algérie in Roubet 1979)

\begin{tabular}{|c|c|c|c|c|c|c|c|c|c|}
\hline \multirow[t]{3}{*}{ DETERMINATION } & \multicolumn{8}{|c|}{$\begin{array}{l}\text { GROTTE CAPÉLETTI: SERIES } \\
\text { D'OCCUPATION NEOLITHIQUES }\end{array}$} & \multirow[t]{2}{*}{ TOTAL } \\
\hline & \multicolumn{2}{|c|}{$1^{\mathrm{re}}$} & \multicolumn{2}{|c|}{$2^{\mathrm{e}}$} & \multicolumn{2}{|c|}{$3^{\mathrm{e}}$} & \multicolumn{2}{|c|}{$4^{\mathrm{e}}$} & \\
\hline & $\mathrm{N}$ & $\%$ & $\mathrm{~N}$ & $\%$ & $\mathrm{~N}$ & $\%$ & $\mathrm{~N}$ & $\%$ & \\
\hline Ovinés et Caprinés & 307 & 89,7 & 3838 & 84,7 & 5459 & 90,4 & 803 & 70,0 & 10407 \\
\hline Bovinés & 25 & 7,3 & 635 & 14,0 & 412 & 6,8 & 283 & 24,7 & 1355 \\
\hline Suinés & 2 & $<1$ & 30 & $<1$ & 124 & 2,0 & 29 & 2,5 & 185 \\
\hline Antilopinés Oryginés & 8 & 2,3 & 9 & $<1$ & 16 & $<1$ & 19 & 1,6 & 52 \\
\hline Canidés & - & - & - & - & 9 & $<1$ & 5 & $<1$ & 14 \\
\hline Viverridés & - & - & 9 & $<1$ & 1 & $<1$ & 2 & $<1$ & 12 \\
\hline Léporidés & - & - & - & - & 1 & $<1$ & - & - & 1 \\
\hline Oiseaux & - & - & 1 & $<1$ & 3 & $<1$ & 3 & $<1$ & 7 \\
\hline Indéterminés & - & - & 6 & $<1$ & 8 & $<1$ & 3 & $<1$ & 17 \\
\hline TOTAUX & 342 & & 4528 & & 6033 & & 1147 & & 12050 \\
\hline
\end{tabular}

Tableau 2. Grotte Capéletti : Instruments exogènes introduits durant la dernière série d'occupation (d'après A. Bachir-Bacha 1996). Légende : astérisque * : documents découverts par J.-B. Capéletti. $S$ : simple ; $\mathrm{D}$ : double ; $\mathrm{B}$ : bouchardage ; $\mathrm{P}$ : polissage.

\begin{tabular}{|c|c|c|c|c|c|c|}
\hline Musée de l'Homme & Références & Roche & Munsell & Dureté & Couleur & Technique \\
\hline Hache épaisse * & $36.1 .2305 \mathrm{~N}$ & Micro-diorite & $5 Y 5 / 1$ & 6 & Gris & $\mathrm{B} / \mathrm{P}$ \\
\hline Hache épaisse * & 36.1 .85 & Diorite & $5 \mathrm{Y} 4 / 2$ & 6 & Vert olive & $\mathrm{B} / \mathrm{P}$ \\
\hline Hache épaisse * & 36.1 .86 & Diorite & $5 \mathrm{Y} 4 / 3$ & 6 & Vert olive & $\mathrm{B} / \mathrm{P}$ \\
\hline Hache épaisse * & 36.1 .87 & Diorite & $7.5 \mathrm{R} 3 / 0$ & 6 & Gris foncé & $\mathrm{B} / \mathrm{P}$ \\
\hline Hache épaisse * & 36.1 .88 & Grès & $5 Y R 6 / 3$ & 7 & Brun-rouge & $\mathrm{B} / \mathrm{P}$ \\
\hline Hache plate & 36.1 .920 & Silex & $7.5 \mathrm{R} 5 / 0$ & 7 & Gris & $\mathrm{B} / \mathrm{P}$ \\
\hline Hache taillée & 36. 1.2084 & Silex & 10YR 6/3 & 7 & Brun pâle & Taillée \\
\hline Herminette S. & 36.1 .82 & Silex & 10YR 6/2 & 7 & Brun clair & $\mathrm{P}$ \\
\hline Herminette S. & 36.1 .84 & Silex & $5 \mathrm{Y} 4 / 4$ & 7 & Vert olive & $\mathrm{P}$ \\
\hline Herminette S. & 36. 1.922 & Serpentine & $7.5 \mathrm{R} 2 / 0$ & 4 & Noir & $\mathrm{P}$ \\
\hline Herminette S. & 36. 1.921 & Serpentine & $7.5 \mathrm{R} 2 / 0$ & 7 & Noir & $\mathrm{P}$ \\
\hline Herminette D.* & 36.1 .81 & Silex & 10YR 5/2 & 7 & Gris brun & $\mathrm{P}$ \\
\hline Herminette D.* & 36.1 .83 & Silex & 10YR 5/2 & 7 & Gris brun & $\mathrm{P}$ \\
\hline Hache épaisse & 36.1.1926 & Diorite & $7.5 \mathrm{R} 3 / 0$ & 7 & Gris foncé & $\mathrm{B} / \mathrm{P}$ \\
\hline Hache épaisse & 36. 1. 1931 & Diorite & $5 \mathrm{Y} 5 / 2$ & 6 & Vert olive & $\mathrm{B}$ \\
\hline Hache plate & 36.1.1987 & Serpentine & $7.5 \mathrm{R} 2 / 0$ & 4 & Noir & $\mathrm{P}$ \\
\hline Hache plate & 36.1.1986 & Schiste & $7.5 \mathrm{R} 3 / 0$ & 6 & Gris foncé & $\mathrm{P}$ \\
\hline Hache plate & 36.1 .2136 & Serpentine & $7.5 \mathrm{R} 2 / 0$ & 5 & Noir & $\mathrm{P}$ \\
\hline Hache plate & 36. 1. 2094 & Serpentine & $7.5 \mathrm{R} 2 / 0$ & 4 & Noir & $\mathrm{P}$ \\
\hline Ciseau & 36.1 .1930 & Quartzite & 10R $5 / 2$ & 7 & Rouge pâle & $\mathrm{B}$ \\
\hline Fragment & 36.1 .2137 & Quartzite & 10YR 4/1 & 7 & Gris foncé & $\mathrm{P}$ \\
\hline
\end{tabular}




\section{Conclusion}

71 Dès lors, c'est le massif tout entier qui devint pour ces bergers et leurs troupeaux un territoire refuge. Les traces et empreintes que bêtes et gens laissèrent sur leur passage sont restées localisées, mais toujours signifiantes. Les bergers néolithiques commencèrent à « humaniser ", à « apprivoiser » une nature majestueuse.

En ouvrant des chemins, en défrichant des passages, en entretenant des pentes et des gués, en mettant en valeur des sources, les pasteurs firent du massif un terroir accueillant. Pour la première fois, une entité sociologique typique, ayant un statut social stable et fort, reconnu ailleurs, vit à l'unisson dans un cadre géographique montagnard, faisant corps avec ses paysages au point de s'y blottir et de l'aménager à la manière d'un « chez soi ».

\section{BIBLIOGRAPHIE}

ALIMEN H., BIÉDA Z., et CASTA L., 1979. « Étude sédimentologique de la Grotte Capéletti » in Roubet C, Économie pastorale préagricole en Algérie orientale. Le Néolithique de tradition capsienne. Exemple:

L'Aurès ». CNRS, Paris, p. 190-197.

BACHIR-BACHA A., 1996. «Réexamen du Néolithique de tradition capsienne, d'après le matériel de la grotte Capéletti (Algérie Orientale) ». Université de Poitiers, Département d'Histoire et d'Archéologie, $516 \mathrm{p}$.

BALLAIS J.-L., MARRE A., ROGNON P., 1979. « Périodes arides du Quaternaire récent et déplacement des sables éoliens dans les Zibans (Algérie) ». Revue de Géol. dyn. et de Géog. phys., vol. 21, fasc. 2, Paris, p. 97-108.

BALLAIS J.-L., ROUBET C, 1981-1982. « Morphogenèse et Préhistoire dans les Aurès ». Revue de Géol. dyn. et de Géog. phys., vol. 23, fasc. 5, p. 375-384.

BALLAIS J.-L., 1984. «Recherches géomorphologiques dans les Aurès Algérie) ». ANRT, Lille, 2 tomes, $626 \mathrm{p}$.

BALLAIS J.-L., 1987. « A.322. Aurès ». Encyclopédie Berbère, XVII, Édisud, p. 1066-1095. Beucher F., 1979. «Étude palynologique de la grotte Capéletti. in Roubet $C$, Économie pastorale préagricole en Algérie orientale. Le Néolithique de tradition capsienne. Exemple : L'Aurès ». CNRS, Paris, p. 419-426.

CAMPS G., 1974. « Les civilisations préhistoriques de l'Afrique du Nord et du Sahara ». Doin, Paris, 373 p. CAMPS G., 1975. « Les industries épipaléolithiques du Maghreb et du Sahara septentrional ». Colloque International d'Aix-en-Provence, sous la dir. de G. Camps (juin 1972), CNRS, p. 83-117.

CAMPS G., 1991. « B.54. Le Bélier à sphéroïdes (Gravures rupestres de l'Afrique du Nord)». Encyclopédie Berbère, IX, Édisud, t. p. 1417-1433.

CAMPS-FABRER H., 1975. « Un gisement Capsien de faciès Sétifien. Médjez II. El-Eulma, (Algérie) ». CNRS, Paris, $448 \mathrm{p}$. 
CARTER P.-L., HIGGS E.S., 1979. « A study of the faunal remains from La grotte Capéletti du Khanguet Si Mohamed Tahar (Aurès, Algérie) » in Roubet C, Economie pastorale, préagricole, en Algérie orientale. Le Néolithique de tradition capsienne. Exemple : l'Aurès ». CNRS, Paris, p. 411-414.

CHANARD E., 1990. « Approche méthodologique des pierres à rainures. Test sur 19 documents de Brézina. Atlas Saharien, Algérie ». Muséum national d'histoire naturelle, DEA, IPH, Paris.

CLUTTON-BROCK J., 1979. « Canids » in Roubet C, Économie pastorale, préagricole, en Algérie orientale. Le Néolithique de tradition capsienne. Exemple : l'Aurès, CNRS, Paris, p. 415-416.

CÔTE M., 1987. « Comment les hommes ont utilisé les piémonts ». Hommage à G. Maurer, C.I.E.M., fasc. 11, Poitiers, p. 221-240. L'auteur étudie notamment le piémont méridional de l'AurèsNémencha.

CôTE M., 1991. « B 53. Bélezma ». Encyclopédie Berbère, IX, Edisud, p. 1415-1417.

COUVERT M., 1969. « Étude de quelques charbons préhistoriques de la grotte Capéletti ». Libyca, XVII, p. 213.217.

FERRÉ B., 1979. « Étude sur l'ensoleillement de l'entrée de la grotte Capéletti » in Roubet C, Économie pastorale, préagricole, en Algérie orientale. Le Néolithique de tradition capsienne. Exemple : l'Aurès, CNRS, Paris, p. 521-546.

GRÉBÉNART D., 1969. « Aïn Naga : Capsien et Néolithique des environs de Messad ». Libyca, XVII, p. 93-197.

GRÉBÉNART D., 1970. « Problèmes du Néolithique près d'Ouled Djellal et de Djelfa : Botma Si Mammar et Safiet Bou Rhenan ». Libyca, XVIII, p. 47-66.

GRÉBÉNART D., 1971. «Vues générales sur le peuplement capsien au Nord des Némencha, secteur de Chéria, Télidjène, et Rass-el-Euch ». Libyca, XIX, p. 171-177.

HACHI S., 1999. «L'Homme de Mechta-Afalou, Cro-Magnon de l'Afrique du Nord durant les 20 derniers millénaires : évolution culturelle et devenir ». Thèse d'Habilitation à Dirigerdes Recherches. Université Paul Valéry, Montpellier III, 1999, 4 volumes.

HACHI S., 2004. Aux origines des arts premiers en Afrique du Nord. Les figurines et les objets modelés en terre cuite de l'abri sous-roche préhistorique d'Afalou, Babors, Algérie. Alger,CNRPAH, n.s., 6.

HACHI S., FRÖHLICH E, GENDRON-BADOU A., LUMLEY H DE., ROUBET C, ABDES-SADOK S., 2002. « Figurines du Paléolithique supérieur en matière minérale plastique cuite d'Afalou Bou Rhummel (Babors, Algérie) ». Premières analyses par spectroscopie Infrarouge. L'Anthropologie, 106, p. 57-97.

HUGOT H.-J., 1963. « Recherches préhistoriques dans l'Ahaggar Nord-Occidental, 1950-1957 ». Mémoire 1, CRAPE, Paris, AMG.

JOLEAUD L., LAFFITTE R., 1934. « Grotte préhistorique du Khanguet Si Mohammed Tahar (Aurès, Algérie) ». Journal de la Société des Africanistes, IV, p. 111-113.

JOLEAUD L., LAFFITTE R., 1934. « Le remplissage d'une grotte préhistorique du Khanguet Si Mohammed Tahar (Aurès, Algérie) ». L'Anthropologie, XLIV, p. 469-471.

LAFFITTE R., 1939. «Étude géologique de l'Aurès ». Bulletin du Service de la Carte Géologique de l'Algérie. $2^{e}$ Série. Stratigraphie. Descriptions régionales, Alger, 484 p., 1 carte h.t.

LARTIGUE LT., 1904. « Monographie de l'Aurès ». Marle-Audrino, Paris, 491 p.

MITTARD A.-D., 1941. « Aperçu des grands traits géographiques de l'Aurès ». Revue de Géog. Alpine, XXIX, Grenoble, p. 557-558. 
PAILLET J.-L., 1979. « Levé topographique de la grotte Capéletti au 1/100 ${ }^{\mathrm{e}}$ » in Roubet C, Économie pastorale, préagricole, en Algérie orientale. Le Néolithique de tradition capsienne. Exemple : l'Aurès, CNRS, Paris, p. 160.

PLU A. ET PORTÈRES R., 1979. « Identification des macro-restes végétaux de la grotte Capéletti » in Roubet C, Économie pastorale, préagricole, en Algérie orientale. Le Néolithique de tradition capsienne. Exemple : l'Aurès, CNRS, Paris, p. 426-448.

PORTÈRES R.,1979. «Présence et utilisation des végétaux identifiés dans la grotte Capéletti » in Roubet C, Économie pastorale, préagricole, en Algérie orientale. Le Néolithique de tradition capsienne. Exemple: l'Aurès, CNRS, Paris, p. 439-448.

ROUBET C, 1966. « Les pendeloques en plaques dermiques de tortue dans le Néolithique de l'Afrique du Nord ». Libyca, XIV, p. 223-259.

ROUBET C, 1968. « Le gisement du Damous el-Ahmar et sa place dans le Néolithique de tradition capsienne » . Travaux du CRAPE, Paris, AMG, $144 \mathrm{p}$.

ROUBET C, 1969. « La grotte Capéletti de Khanguet Si Mohamed Tahar, Aurès, Algérie. Étude préliminaire ». Libyca, XVII, p. 203-211.

ROUBET C, 1979. «Économie pastorale préagricole en Algérie orientale : le Néolithique de tradition capsienne. Exemple L'Aurès, CNRS, Études d'Antiquités Africaines, Paris, 595 p.

ROUBET C, CARTER P. L., 1984. « Origine de la transhumance ovine dans le Maghreb oriental ». in The Deya Conference of Prehistory : Early settlement in Western Mediterranean Islands and the peripheral areas. Ed. by W. H. Waldren and coll. BAR, International Séries, $\mathrm{n}^{\circ} 229$, p. 239-248.

ROUBET C, 1985. « Transhumance ovine dans l'Aurès depuis le Ve millénaire B. C ». Studi di Paletnologia in onore di Salvato M. Puglisi. Ed. By M. Liverani, A. Palmieri, R. Peroni, Università di Roma, La Sapienza, p. 421-423.

ROUBET C, 1995. « Le « statut de berger ». Étude des communautés pastorales de l'Atlas du Maghreb oriental ». Document interne. Muséum national d'histoire naturelle, Paris, 25 pages.

ROUBET C, 2001a. «Le Néolithique de Tradition Capsienne sensu stricto ». Encyclopedia of Prehistory. Volume 1: Africa, 10 entrées, in Outlines of Archaeological Traditions. The Human Relations Area Files, Ed. by P.-N. Peregrine and M. Ember, Kluwer Académie, Plenum Publishers, p. 197-219.

ROUBET C, HACHI S., 2001b. « Les sépultures de Méchta-Afalou, Cro-Magnon de l'Afrique du Nord : approche culturelle à partir de nouvelles découvertes ». Colloque International du Val-deMarne : L'Identité humaine en question, sous la dir. de Ph. Andrieux, D. Hadjouis et A. DambricourtMalassé, Artcom, Paris, p. 225-245.

ROUBET C, 2003a. «Économie pastorale préagricole dans l'Aurès Néolithique. Gestion et organisation des cheptels en environnement atlasique ». Archéologia, Dijon, n²82, pp. 62-71.

ROUBET C, 2003b. « Statut de berger des communautés atlasiques néolithisées du Maghreb oriental, dès $7000 \mathrm{BP} »$. ' 'Anthropologie, 107, pp. 393-442.

VAUFREY R., 1939. «L'Art rupestre nord-africain ». Archives de l'Inst. de Paléontologie Humaine XX, Paris, Masson, $128 \mathrm{p}$.

\section{INDEX}

Mots-clés : Aurès, Néolithique, Pastoralisme, Préhistoire 